\title{
The nutritional management of surgical patients: enhanced recovery after surgery
}

\author{
Kenneth C. H. Fearon* and Rachel Luff \\ Department of Clinical and Surgical Sciences (Surgery), School of Clinical Sciences and Community Health, The University of \\ Edinburgh, Room F3307, The Royal Infirmary of Edinburgh, 51 Little France Crescent, Edinburgh EH16 45A, UK
}

\begin{abstract}
Malnutrition has long been recognised as a risk factor for post-operative morbidity and mortality. Traditional metabolic and nutritional care of patients undergoing major elective surgery has emphasised pre-operative fasting and re-introduction of oral nutrition 3-5d after surgery. Attempts to attenuate the consequent nutritional deficit and to influence post-operative morbidity and mortality have included parenteral, enteral and oral sip feeding. Recent studies have emphasised that an enhanced rate of recovery can be achieved by a multi-modal approach focused on modulating the metabolic status of the patient before (e.g. carbohydrate and fluid loading), during (e.g. epidural anaesthesia) and after (e.g. early oral feeding) surgery. Using such an approach preliminary results on patients undergoing elective colo-rectal surgery indicate a significant reduction in hospital stay (traditional care, $n 48$, median stay $10 \mathrm{~d} v$. enhanced recovery programme, $n 33$, median stay $7 \mathrm{~d} ; P<0.01)$ can be achieved. Such findings emphasise the potential role of multi-modal care programmes in the promotion of early recovery from major surgical trauma.
\end{abstract}

Pre-operative fasting: Post-operative nutritional support: Enhanced recovery after surgery

The aim of the present article is to review the nutritional management of the surgical patient. In particular, the rationale and nature of nutritional support within the context of traditional peri-operative care will be compared with that for enhanced recovery following surgery.

\section{Nutritional status and surgical outcome}

The reasons why nutritional status is considered to be an important determinant of surgical outcome can be traced back to the 1930s and the studies of Studley (1936). He demonstrated in patients undergoing partial gastrectomy for complicated peptic ulcer disease that those who had lost $<20 \%$ of their pre-illness stable weight had an operative mortality of approximately $3 \%$ whereas those with $>20 \%$ weight loss had an operative mortality of approximately $30 \%$. It might be argued that more modern surgery with prophylactic antibiotics, better anaesthesia, improved suture materials and optimal physiotherapy might avoid such a high rate of post-operative complications in malnourished patients. However, more recent studies still show a similar relationship. Hill (1994), for example, used neutronactivation body composition analysis to determine total body $\mathrm{N}$ and thus define those patients with or without a marked protein deficit. The study involved $>100$ patients undergoing gastrointestinal tract resection. There was a less dramatic but still marked increase in both major complications and the duration of post-operative stay in those patients with marked whole-body protein depletion. Clearly, it is difficult to separate post-operative morbidity related to malnutrition per se $v$. that related primarily to the patients' underlying pathology (which just happens to have induced malnutrition). Nevertheless, such studies have formed the basis for using nutritional support to try to reduce postoperative morbidity and mortality.

\section{Parenteral nutrition}

Unfortunately, throughout the 1980s and into the 1990s nutritional support came to be regarded as a panacea for all surgical ills. In particular, numerous trials of perioperative total parenteral nutrition were undertaken in 
cohorts of patients who were not specifically malnourished. Heyland et al. (1998) have recently undertaken a metaanalysis and, not surprisingly, concluded that peri-operative parenteral nutrition given to patients undergoing mainly major upper gastrointestinal cancer surgery has no influence on post-operative major complications or mortality.

\section{Enteral nutrition}

During the 1990s there was a shift in emphasis from parenteral nutrition to enteral nutrition, particularly for the care of the critically ill. The rationale for a change in emphasis included the fact that enteral feeding is more physiological, is associated with fewer complications and is also cheaper. However, for the post-operative patient with a gastrointestinal anastomosis, there is the anxiety that filling the gastrointestinal tract with enteral feed in the early postoperative period might increase anastomotic leak rate and might also be associated with an increased incidence of aspiration pneumonia.

Following traditional open abdominal surgery patients frequently experience a period of gastrointestinal paralysis (ileus). The small bowel is the first section of the gastrointestinal tract to regain normal motility, and this factor has been the rationale for the use of naso-jejunal or needlecatheter jejunostomy for the provision of early enteral feeding in post-operative patients undergoing major abdominal surgery. That this method of feeding can be undertaken in a safe manner has been demonstrated by a number of different research groups. For example, in a consecutive series of 500 patients Sarr (1999) demonstrated that only three patients had major complications related to their feeding jejunostomy. Moreover, using the jejunostomy route $>90 \%$ of patients achieved their goals for full enteral nutritional support and fluid and electrolyte balance by the fourth post-operative day. However, as in all medical or surgical interventions it is imperative to carry these out with minimum morbidity and mortality. Smith et al. (1985) reported on a series of fifty patients undergoing major upper gastrointestinal resection for cancer who were randomised to post-operative jejunostomy feeding or conventional intravenous fluids. Mean hospital stay was significantly $(P<0.05)$ prolonged in the group undergoing enteral feeding. Moreover, there were twenty catheterrelated complications and one death due to leakage from a catheter into the peritoneal cavity. Such studies emphasise that any potential benefit from nutritional support can be overwhelmed in the presence of complications related to failures of technique.

In terms of nutritional status patients with major burns appear to benefit from early enteral feeding, but this form of nutritional support is not associated with a reduction in morbidity or mortality (Gottschlich et al. 2002). When compared with total parenteral nutrition, early enteral feeding in patients with major abdominal trauma results in a reduction in septic morbidity (Kudsk et al. 1992a,b). However, it has been suggested that such an apparent benefit is related more to the adverse effects of overfeeding with total parenteral nutrition than to any true benefit from enteral feeding per se (Jeejeebhoy, 2001). For patients in the intensive care unit the situation is complex. However, the overall consensus is that a shift from parenteral nutrition to enteral nutrition has been of major benefit over the last 10 years. In relation to the routine post-operative surgical patient it is more difficult to achieve a consensus. A recent meta-analysis has emphasised a potential reduction in postoperative infectious complications in patients given enteral feeding with an immunomodulatory regimen (Heys et al. 1999). However, in one of the largest single-centre studies (Heslin et al. 1997a,b) 195 patients with oesophageal, gastric, pancreatic or bile duct cancer were randomised to post-operative early enteral feeding with an immunomodulatory regimen via a jejunostomy tube $v$. standard intravenous fluids before commencement of oral diet. There were no differences in major complications, length of stay or post-operative mortality. Thus, the role of routine placement of a feeding jejunostomy in patients undergoing major upper gastrointestinal surgery is really only justified on the basis of the usefulness of this route for the feeding of patients who go on to develop intra-abdominal complications, rather than in the prevention of complications in the first instance. Moreover, in the absence of routine measures to improve gastrointestinal function in the immediate post-operative period it has been demonstrated that early post-operative enteral feeding results in impaired respiratory mechanics and decreased mobility (Watters et al. 1997a,b).

It is now recognised that for the vast majority of patients post-operative complications relate more to pre-operative morbidity and the technical aspects of surgery than to nutritional status per se. In particular, Pettigrew \& Hill (1987) demonstrated in a large cohort of patients undergoing abdominal surgery that the best predictor of post-operative complications was the post-operative assessment by the surgeon of how well the surgery had gone. The development of more modern anaesthetic agents and the frequent use of epidural anaesthesia (Rodgers et al. 2000) have probably further attenuated the importance of the nutritional status of the patient in relation to postoperative morbidity and mortality. In summary, in a modern context it seems less realistic to expect that routine nutritional support might influence post-operative outcome in terms of major morbidity and mortality. More precisely, there has been a shift in emphasis towards the use of nutritional support to influence the rate and quality of recovery of the patient.

\section{Traditional post-operative care}

Conventional post-operative care has emphasised resting the gastrointestinal tract until full recovery of function occurs. Patients are routinely fasted overnight pre-operatively and frequently receive bowel preparation that can cause both dehydration and fluid and electrolyte abnormalities. Recent evidence has suggested it is entirely safe to allow intake of clear fluids up to $2 \mathrm{~h}$ before anaesthesia in most patients (Fasting et al. 1998). It has also been suggested that routine fluid and carbohydrate loading $2 \mathrm{~h}$ before surgery can attenuate post-operative insulin resistance and improve patient well-being (Ljungqvist et al. 2002). Moreover, 
bowel preparation may not be necessary at all (Santos et al. 1994). Conventional pre-operative medication and anaesthesia can often leave the patient sedated for several hours following surgery and therefore unable to sit up and take fluids or diet. Opiate analgesia is used frequently and this practice can contribute to post-operative sedation and also increase the incidence of post-operative ileus, nausea and vomiting. Patients frequently have a 'nil by mouth' sign posted above their bed for 3-5 d in the post-operative period, and during this time they receive maintenance fluids via the intravenous route. Recent evidence has suggested that excess intravenous fluids (and in particular saline $(9 \mathrm{~g}$ $\mathrm{NaCl} / \mathrm{l})$ ) may contribute to post-operative gastrointestinal tract dysfunction, prolong post-operative stay (Lobo et al. 2002) and adversely affect overall clinical outcome (Frost et al. 2001). Finally, patients also undergo enforced bedrest, not only as a part of traditional care, but also because of the presence of drips, catheters and drains.

Within this traditional pattern of post-operative care patients are thus subject to a period of starvation and immobilisation that may last $\leq 1$ week. In order to combat the nutritional consequences of such starvation oral supplements have been tested in order to try to enhance normal food intake following recommencement of oral nutrition. Such studies have demonstrated that oral supplements can attenuate post-operative weight loss (Beattie et al. 2000). However, recovery to pre-operative levels of nutritional status may take many weeks or months. Prevention is surely the better policy.

\section{'Enhanced recovery' post-operative care}

A more fundamental shift in terms of post-operative care has been to consider enhancing the overall recovery rate of patients following open abdominal surgery. This approach has been pioneered by Henrik Kehlet, in Copenhagen, Denmark, who suggested that the normal decline in functional status that may occur over a period of weeks in the post-operative period could be shortened to a matter of

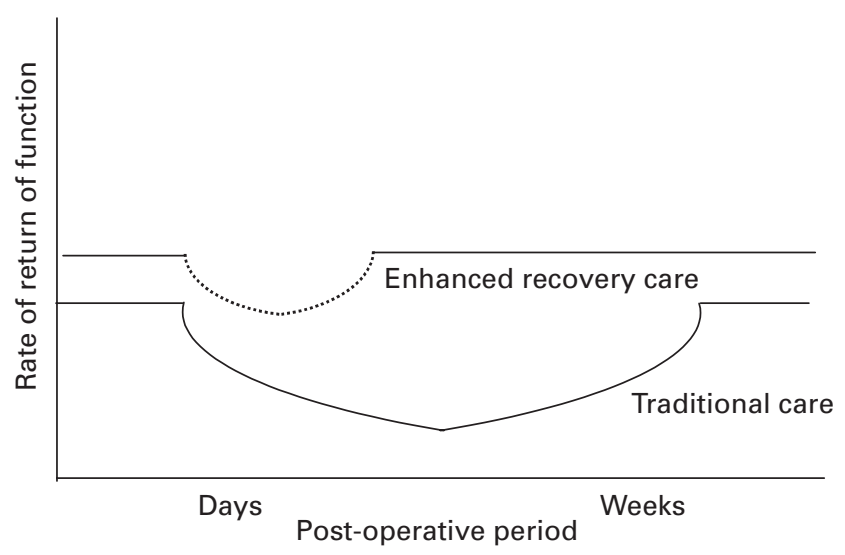

Fig. 1. Different patterns of recovery following 'traditional' $v$. 'enhanced recovery' post-operative care. days by the use of multi-modal intervention (Fig. 1). He has focused on post-operative pain, gut function and mobility. These areas are seen as the main domains that delay time of discharge. Patients can return home if their pain is adequately controlled with simple oral analgesia, if they are able to take sufficient fluids and nutrition to maintain homeostasis, and if they are mobile enough to go to the toilet etc. The interventions to address such issues include optimisation of post-operative pain relief with epidural anaesthesia. The latter is carried on for $48-72 \mathrm{~h}$ and allows patients to be virtually pain free during this period. Epidural anaesthesia and analgesia reduce both post-operative ileus and the metabolic response to injury by blocking visceral sympathetic and parasympathetic pathways (Holte \& Kehlet, 2002). The use of early oral enteral nutrition is further encouraged by avoidance of both sedation in the post-operative period and the routine use of opiate analgesia. Patients are mobilised early on the basis of adequate pain relief and a well-defined nursing care pathway.

The success of this approach has been shown in studies examining the recovery of patients following colo-rectal resection (Basse et al. 2000). In a consecutive series of sixty patients discharge was planned for $48 \mathrm{~h}$ following surgery (rather than the more conventional 10-14 d). The median age of the patients was 74 years and at least one-third of patients had marked comorbidity. Thirty-two of the sixty patients went home within $48 \mathrm{~h}$, indicating that with an accelerated stay programme it is possible to discharge the majority of patients undergoing colo-rectal surgery above the pelvic brim within a very short time frame.

As part of the Enhanced Recovery After Surgery Study Group the possibility of establishing such an approach in multiple countries throughout Europe has been examined. In contrast to the normal policy of pre- and post-operative starvation it has been demonstrated in the Colorectal Unit in Edinburgh, UK that the majority of patients undergoing major pelvic (anterior resection) surgery are able to take preoperative fluid and carbohydrate loading up to $2 \mathrm{~h}$ before going to surgery, and that at least a proportion are able to commence oral fluids and oral feeds on the night of operation and have their intravenous fluids discontinued on their first or second post-operative day (Fig. 2). Moreover, hospital stay has been reduced from approximately $10 \mathrm{~d}$ with traditional care to $7 \mathrm{~d}$ with the enhanced recovery programme (Fig. 3). Clearly, this remarkable change in rate of recovery cannot be ascribed entirely to the nutritional component of the programme. It will take many future studies to define precisely the role of each individual component in relation to patient's rate of recovery.

\section{Conclusion}

Although artificial enteral or parenteral nutritional support is not generally indicated for elective abdominal surgery, the traditional approach of starving the patient undergoing major abdominal surgery is now strongly under question. It appears that if a multi-modal care pathway is adopted marked oral nutrition and fluid intake can be achieved in the immediate peri-operative period and the overall rate of recovery can be enhanced. 


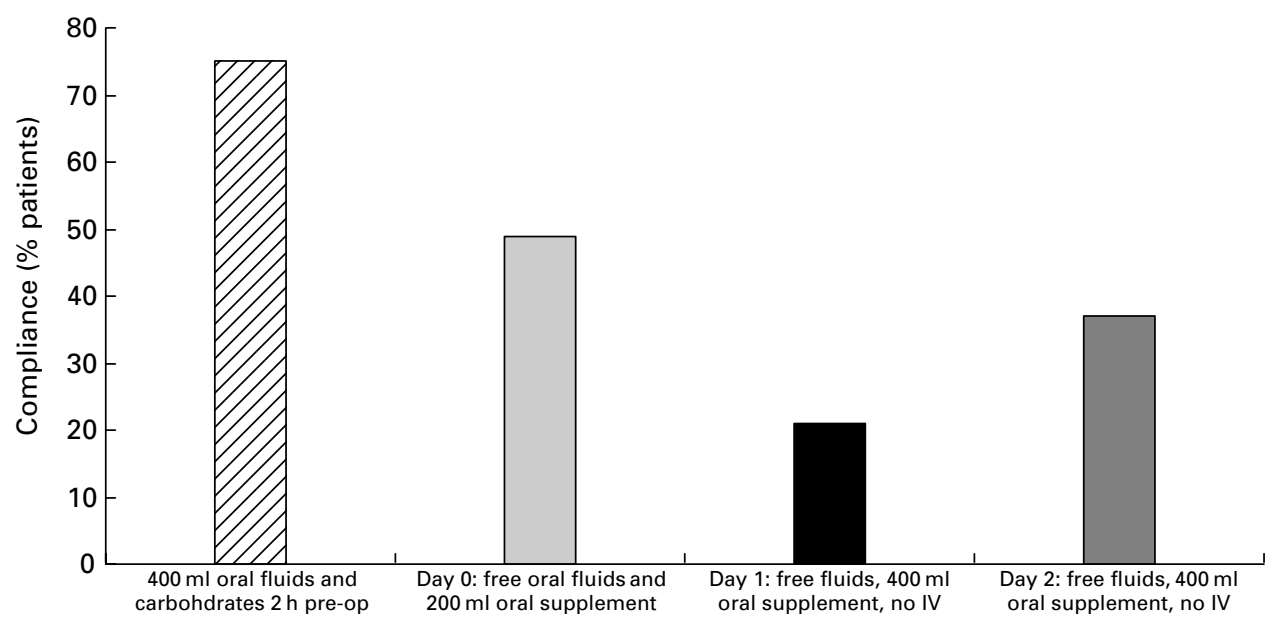

Fig. 2. Nutrition and fluid management in patients $(n 33)$ undergoing colo-rectal (anterior) resection. Patients were subject to a multi-modal

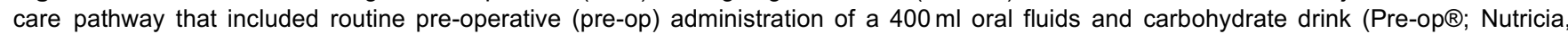
Trowbridge, Wilts., UK; mas) and free oral fluids and $200-400 \mathrm{ml}$ of a whole-protein oral sip feed (Fortisip $\AA$; Nutricia; ( $\mathbf{\square})$ from the day of surgery onwards. Compliance is documented on post-operative day $1(\square)$ and $2(\square)$ for patients who were not only able to consume $400 \mathrm{ml}$ of the sip feed but who were able to take sufficient fluids overall to allow discontinuation of intravenous fluids (no IV). (KCH Fearon and R Luff on behalf of the Enhanced Recovery After Surgery Group, unpublished results).

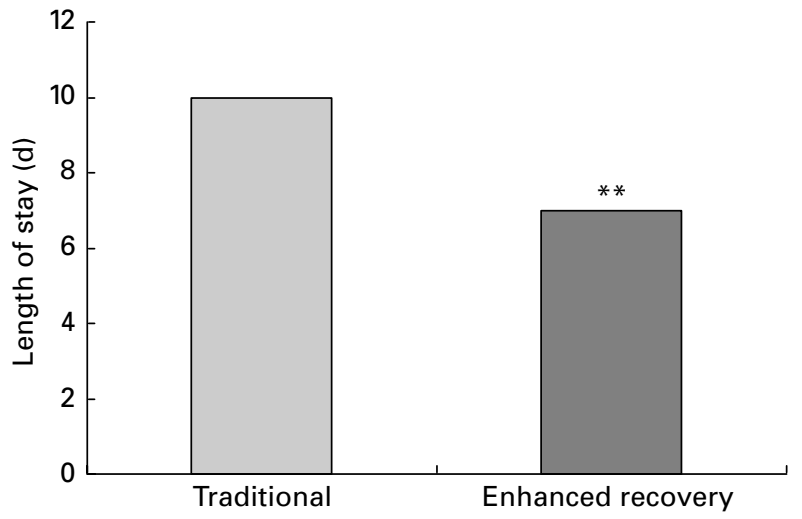

Fig. 3. Length of hospital stay in a consecutive series of patients undergoing colo-rectal (anterior) resection and following either a traditional peri-operative care pathway $(n 48)$ or an enhanced recovery after surgery pathway $(n 33)$. Median values were significantly different from those for the traditional treatment group (Mann-Whitney $U$ test): ${ }^{* *} P<0 \cdot 01$. (KCH Fearon and $\mathrm{R}$ Luff on behalf of the Enhanced Recovery After Surgery Group, unpublished results.)

\section{Acknowledgements}

The Enhanced Recovery After Surgery Group is supported by a study grant from Nutricia.

\section{References}

Basse L, Hjort Jakobsen D, Billesbolle P, Werner M \& Kehlet H (2000) A clinical pathway to accelerate recovery after colonic resection. Annals of Surgery 232, 51-57.

Beattie AH, Prach AT, Baxter JP \& Pennington CR (2000) A randomised controlled trial evaluating the use of enteral nutritional supplements postoperatively in malnourished surgical patients. Gut 46, 813-818.
Fasting S, Soreide E \& Raeder JC (1998) Changing preoperative fasting policies. Impact of a national consensus. Acta Anaesthesiologica Scandinavica 42, 1188-1191.

Frost A, Wakefield CH, Sengupta F \& Fearon K (2001) Relationship between fluid administrations and outcome in colorectal surgery. Proceedings of the Nutrition Society 60, 113A.

Gottschlich MM, Jenkins ME, Mayes T, Khoury J, Kagan RJ \& Warden GD (2000) An evaluation of the safety of early vs delayed enteral support and effects on clinical, nutritional, and endocrine outcomes after severe burns. Journal of Burn Care and Rehabilitation 23, 401-415.

Heslin MJ, Latkany L, Leung D, Brooks AD, Hochwald SN, Pisters PW, Shike M \& Brennan MF (1997a) A prospective, randomized trial of early enteral feeding after resection of upper gastrointestinal malignancy. Annals of Surgery 226, 567-577.

Heslin MJ, Latkany L, Leung D, Brooks AD, Hochwald SN, Pisters PW, Shike M \& Brennan MF (1997b) A prospective, randomized trial of early enteral feeding after resection of upper gastrointestinal malignancy: discussion. Annals of Surgery 226, $577-580$

Heyland DK, MacDonald S, Keefe L \& Drover JW (1998) Total parenteral nutrition in the critically ill patient: a metaanalysis. Journal of the American Medical Association 280, 2013-2019.

Heys SD, Walker LG, Smith I \& Eremin O (1999) Enteral nutritional supplementation with key nutrients in patients with critical illness and cancer: a meta-analysis of randomized controlled clinical trials. Annals of Surgery 229, 467-477.

Hill GL (1994) Changes in body composition and outcome in surgical patients. Clinical Nutrition 13, 331-340.

Holte K \& Kehlet H (2002) Epidural anaesthesia and analgesia effects on surgical stress responses and implications for postoperative nutrition. Clinical Nutrition 21, 199-206.

Jeejeebhoy KN (2001) Enteral and parenteral nutrition: evidence-based approach. Proceedings of the Nutrition Society 60, 399-402.

Kudsk KA, Croce MA, Fabian TC, Minard G, Tolley EA, Poret HA, Kuhl MR \& Brown RO (1992a) Enteral versus parenteral feeding. Effects on septic morbidity after blunt and penetrating abdominal trauma. Annals of Surgery 215, 503-511. 
Kudsk KA, Croce MA, Fabian TC, Minard G, Tolley EA, Poret HA, Kuhl MR \& Brown RO (1992b) Enteral versus parenteral feeding. Effects on septic morbidity after blunt and penetrating abdominal trauma: discussion. Annals of Surgery 215, 511-513.

Ljungqvist O, Nygren J \& Thorell A (2002) Modulation of postoperative insulin resistance by pre-operative carbohydrate loading. Proceedings of the Nutrition Society 61, 329-335.

Lobo DN, Bostock KA, Neal KR, Perkins AC, Rowlands BJ \& Allison SP (2002) Effect of salt and water balance on recovery of gastrointestinal function after elective colonic resection: a randomised controlled trial. Lancet 359, 1812-1818.

Pettigrew RA \& Hill GL (1986) Indicators of surgical risk and clinical judgement. British Journal of Surgery 73, 47-51.

Rodgers A, Walker N, Schug S, McKee A, Kehlet H, van Zundert A, Sage D, Futter M, Saville G, Clark T \& MacMahon S (2002) Reduction of postoperative mortality and morbidity with epidural or spinal anaesthesia: results from overview of randomised trials. British Medical Journal 321, 1493.

Santos JC Jr, Batista J, Sirimarco MT, Guimaraes AS \& Levy CE (1994) Prospective randomized trial of mechanical bowel preparation in patients undergoing elective colorectal surgery. British Journal of Surgery 81, 1673-1676.

Sarr MG (1999) Appropriate use, complications and advantages demonstrated in 500 consecutive needle catheter jejunostomies. British Journal of Surgery 86, 557-561.

Smith RC, Hartemink RJ, Hollinshead JW \& Gillett DJ (1985) Fine bore jejunostomy feeding following major abdominal surgery: a controlled randomized clinical trial. British Journal of Surgery 72, 458-461.

Studley HO (1936) Percentage of weight loss. A basic indicator of surgical risk in patients with chronic peptic ulcer. Journal of the American Medical Association 106, 458-460.

Watters JM, Kirkpatrick SM, Norris SB, Shamji FM \& Wells GA (1997a) Immediate postoperative enteral feeding results in impaired respiratory mechanics and decreased mobility. Annals of Surgery 226, 369-377.

Watters JM, Kirkpatrick SM, Norris SB, Shamji FM \& Wells GA (1997b) Immediate postoperative enteral feeding results in impaired respiratory mechanics and decreased mobility: discussion. Annals of Surgery 226, 377-380. 